INPLASY

PROTOCOL

To cite: Zhao et al. Efficacy and Safety of Fecal Microbiota Transplantation for Treatment of Ulcerative Colitis Patients: A Systematic Review and MetaAnalysis. Inplasy protocol 202060099. doi:

10.37766/inplasy2020.6.0099

Received: 26 June 2020

Published: 26 June 2020

Corresponding author: Yuqiang Nie

eynieyuqiang@scut.edu.cn

Author Affiliation:

Guangzhou First People's

Hospital

Support: National Natural Science.

Review Stage at time of this submission: Piloting of the study selection process.

Conflicts of interest: None declared.

\section{Efficacy and Safety of Fecal Microbiota Transplantation for Treatment of Ulcerative Colitis Patients: A Systematic Review and Meta-Analysis}

Zhao, HL; Chen, SZ2; Xu, HM³; He, J4; Zhou, YL5; Nie, YQ6.

Review question / Objective: To assess the effect of donor selection, stool procedures, pretreatment with antibiotics on the efficacy and safety of fecal microbiota transplantation (FMT) in the treatment of ulcerative colitis (UC).

Condition being studied: A recent systematic review of FMT in application to treat UC identified 41 studies, but only included 4 random controlled trials (RCTs), 4 controlled cohort studies, and the rest were uncontrolled cohort studies and case series. Since the completion of that review, the number of available studies has more than doubled, including the publication of the latest 3 RCTs of FMT in UC. Furthermore, serious adverse events following FMT in patients with established UC have been reported in recent research. Even though the cumulative research has increased, few endeavors have analyzed aspects of UC and FMT and how these relate to donor selection, stool type, method of FMT delivery, stool total dosages, and whether or not patients were pretreated with courses of any antibiotics.

INPLASY registration number: This protocol was registered with the International Platform of Registered Systematic Review and Meta-Analysis Protocols (INPLASY) on 26 June 2020 and was last updated on 26 June 2020 (registration number INPLASY202060099).

\section{INTRODUCTION}

Review question / Objective: To assess the effect of donor selection, stool procedures, pretreatment with antibiotics on the efficacy and safety of fecal microbiota transplantation (FMT) in the treatment of ulcerative colitis (UC).
Condition being studied: A recent systematic review of FMT in application to treat UC identified 41 studies, but only included 4 random controlled trials (RCTs), 4 controlled cohort studies, and the rest were uncontrolled cohort studies and case series. Since the completion of that review, the number of available studies has more than doubled, including the publication of 
the latest 3 RCTs of FMT in UC. Furthermore, serious adverse events following FMT in patients with established UC have been reported in recent research. Even though the cumulative research has increased, few endeavors have analyzed aspects of UC and FMT and how these relate to donor selection, stool type, method of FMT delivery, stool total dosages, and whether or not patients were pretreated with courses of any antibiotics.

\section{METHODS}

Participant or population: Eligibility criteria were determined by the authors of this study and included measures of clinical efficacy and/or safety of FMT applied for the treatment of UC in human participants. UC patients were treated with FMT intervention therapy, fecal microbiota was required to have been derived from feces of healthy donors, and FMT was applied via any delivery modality. Adults or pediatric participants confirmed with a diagnosis of UC were eligible for inclusion.

Intervention: FMT intervention therapy.

Comparator: UC patients that did not receive FMT treatment.

Study designs to be included: Research designs which employed cohort-based studies and randomized controlled trials were implemented as inclusion criteria.

Eligibility criteria: Eligibility criteria were determined by the authors of this study and included measures of clinical efficacy and/ or safety of FMT applied for the treatment of UC in human participants. UC patients were treated with FMT intervention therapy, fecal microbiota was required to have been derived from feces of healthy donors, and FMT was applied via any delivery modality.

Information sources: Our systematic-based literature search was performed using data available from four electronic databases including PubMed, Web of Science, Embase, and the Cochrane Library and searched without any restriction to language-based results.
Main outcome(s): FMT appears to have been used as an effective and safe treatment to induce remission of active UC.

Quality assessment / Risk of bias analysis: Measures of quality and bias of cohortbased studies were assessed using the Newcastle-Ottawa Scale (NOS), and by using a standard 9-point scale. An adjusted the 6-point scale is evaluated cohort studies lacked a control group. The Cochrane risk of bias score was used to evaluate RCTs.

Strategy of data synthesis: For metaanalyses including uncontrolled cohort studies, the effect size refers to pooled estimates of the proportions of patients that achieved efficacy from FMT-based treatments. For meta-analyses including both RCTs and controlled cohort studies, we calculated measures for pooled odd ratios (P-ORs) and $95 \%$ confidence intervals (Cls).

Subgroup analysis: Meta-subgroup analysis of donor selection, stool type, FMT delivery, FMT total dosage, and pretreatment with antibiotics.

Sensibility analysis: NA.

Country(ies) involved: Australia, Netherlands, Japan, Canada and China.

Keywords: fecal microbiota transplantation; ulcerative colitis; donor selection; stool administration; pretreatment with antibiotics.

Contributions of each author:

Author 1 - Hailan Zhao - HLZ designed the study, collected the data, and wrote the manuscript.

Author 2 - Chen, SZ.

Author 3 - Xu, HM.

Author 4 - He, J.

Author 5 - Zhou, YL.

Author 6 - Nie, YQ. 